\title{
Embryonal prostatic rhabdomyosarcomas disguised presentation in an adolescent male: a case report Nishith K Singh ${ }^{1}$, Malatesha Gangappa ${ }^{2}$, Vineet Gupta ${ }^{3}$ and Anant Mohan ${ }^{4}$
}

\author{
Addresses: ${ }^{1}$ Department of Internal Medicine, Southern Illinois University School of Medicine, Springfield, IL, 62794, USA \\ ${ }^{2}$ Department of Medicine, Berkshire Medical Center, Pittsfield, MA, 01201, USA \\ ${ }^{3}$ Department of Medicine, UPMC Mercy Hospital, Pittsburgh, PA, 15219, USA \\ ${ }^{4}$ Department of Internal Medicine, All India Institute of Medical Sciences, New Delhi, 110029, India \\ Email: NKS - nishith_singh2007@yahoo.com; MG - malateshag@gmail.com; VG* - vineet_gsvm@yahoo.com; \\ AM - anant_mohan@rediffmail.com \\ * Corresponding author
}

Received: 30 April 2009 Accepted: 14 August 2009 Published: 26 August 2009

Cases Journal 2009, 2:7546 doi: 10.4076/1757-1626-2-7546

This article is available from: http://casesjournal.com/casesjournal/article/view/7546

(c) 2009 Singh et al.; licensee Cases Network Ltd.

This is an Open Access article distributed under the terms of the Creative Commons Attribution License (http://creativecommons.org/licenses/by/3.0), which permits unrestricted use, distribution, and reproduction in any medium, provided the original work is properly cited.

\begin{abstract}
Primary rhabdomyosarcoma of the prostate is a malignancy primarily of pediatric age group with most cases seen during infancy and childhood. Majority present with urinary symptoms but the case we report here presented at 16 years of age and had unusually nonspecific and temporally protracted nature of symptoms prior to the presentation. This remarkable unusualness lead to a delay in presentation and contributed to an eventually unfavorable outcome.
\end{abstract}

\section{Introduction}

Primary embryonal prostatic rhabdomyosarcoma (EPRM) is an uncommon tumor and most cases, as reported in the past, present early in age and with predominant lower urinary tract symptoms $[1,2]$. We report a case which is unusual for its conspicuous absence of urinary symptoms or lymphadenopathy in spite of the widespread disease at presentation. The relatively nonspecific constitutional and protracted complaints of fever and polyarthralgias masquerading like that of a systemic illness, lead to a delayed presentation and poor prognosis for the patient.

\section{Case presentation}

A 16-year-old Asian-Indian male student presented with progressively worsening dyspnea on exertion and new onset skin nodules over past one week. He has had polyarthralgias and low grade fever of two months duration. Past and personal history was unremarkable for any significant illness, allergy or high-risk behavior. Patient had received broad spectrum antibiotics prior to the admission for his constitutional symptoms without any clinical benefit. On examination the patient was febrile, pale, normotensive with sinus tachycardia, and had multiple non-tender subcutaneous nodules, two to four centimeters in size, predominantly on the scalp, face and upper trunk. He also had scattered crackles all over the chest, mild splenomegaly and non-tender prostate enlargement. Investigations showed normocytic normochromic anemia, hypercalcemia and hyperuricemia. Routine urinalysis was normal. No laboratory evidence of tuberculosis, immuno-compromised state or an autoimmune disorder was observed. Chest imaging (Figure 1) showed multiple bilateral nodular densities. Patient was hydrated, transfused blood products and put on an empirical broad 


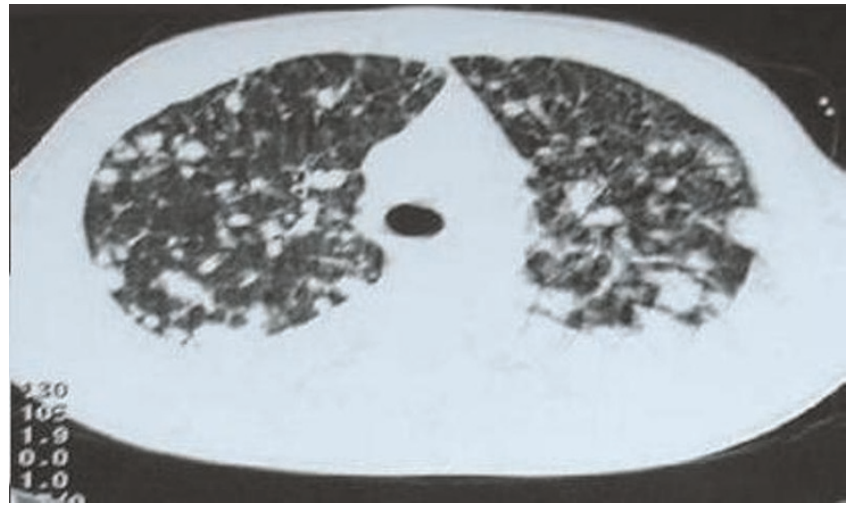

Figure I. CECT chest (lung window) shows multiple varying nodules in both lungs.

spectrum antibiotic cover pending blood and urine culture reports which eventually turned out to be sterile. Computed tomography of abdomen unveiled an incidental, $6 \times 5 \times 5 \mathrm{~cm}$ homogenous soft tissue mass rising from prostrate. The prostate specific antigen (PSA) and prostatic acid phosphatase (PAP) were within normal limits. Trucut biopsy (of forehead nodule) was done; the microscopy and immuno-histochemistry suggested an embryonal rhabdomyosarcoma (Figure 2). Patient subsequently was found to have multiple osteolytic lesions in the skull,

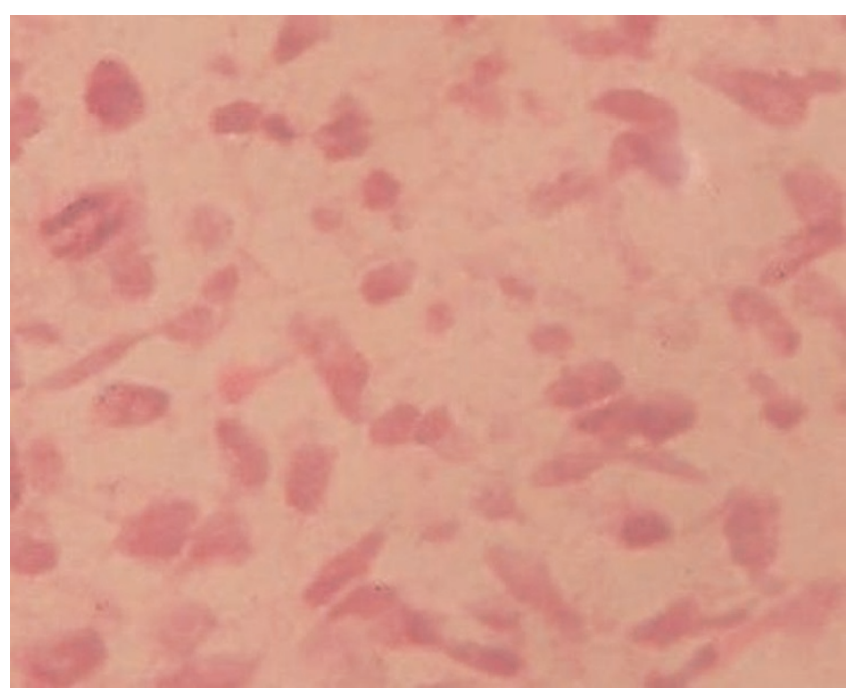

Figure 2. (H/E-400X) showing a malignant small round cell tumor with frequent mitosis and there is no evidence of any differentiation. Immunohistochemistry reveals focal cytoplasmic positivity for desmin and negativity for leukocyte common antigen, cytokeratin, chromogranin, MIC-2 and S-I00 protein. The histological and histochemistry findings are compatible with undifferentiated rhabdomyosarcoma. pelvis and proximal long bones. In view of a metastatic prostatic embryonal rhabdomyosarcoma the patient was started on vincristine, adriamycin and cyclophosphamide regimen. After a transient initial clinical response the patient succumbed to complications of widespread disease after 3 weeks. An autopsy could not be consented for by the patient's relatives.

\section{Discussion}

Embryonal prostatic rhabdomyosarcoma that occurs predominantly in male infants and children (median age of 5 years) is a rare and highly malignant tumour. Only sporadic cases have been reported in non-pediatric age group $[1,2,3]$.

Characteristic features of EPRM published in literature include rapidly progressive obstructive urinary symptoms, smooth and firm enlargement of prostate on digital rectal examination, occasional suprapubic mass, regional lymph node spread, respiratory symptoms due to metastasis to lungs, osteoclastic bone metastases, and normal prostatic acid phosphates and prostate specific antigen levels $[1,2,3]$. The case presented here is atypical in terms of its inconspicuous presentation mimicking a systemic inflammatory response and in the authors' best knowledge has not been reported before. Although primary site in prostate is a favorable prognostic sign for rhabdomyosarcomas, the presence of detectable metastases, tumor size $>5$ centimeters and age $>10$ years at presentation were the associated poor prognostic features in our patient which explains the unfortunate outcome [4]. Tumor debulking (excising $\geq 50 \%$ of tumor) followed by chemotherapy has improved overall survival rates to 75\% in EPRM patients, but our patient had distant detectable spread at the time of presentation which precluded any form of surgical intervention [5].

\section{Conclusion}

Embryonal rhabdomyosarcoma of prostate in non-pediatric age group may have non specific presentation. It is prudent to have a high index of suspicion in diagnosing such cases early to avert grave prognosis.

\section{Abbreviations}

EPRM, embryonal prostatic rhabdomyosarcoma; PAP, prostatic acid phosphatase; PSA, prostate specific antigen.

\section{Consent}

Written informed consent for publication of this case report could not be obtained. The patient died and the next of kin were lost to follow up. Extensive attempts made to obtain consent proved futile. Anonymity of the patient has been thoroughly maintained in this case report. We would not expect the patient's family to object to publication. 


\section{Competing interests}

The authors declare that they have no competing interests.

\section{Authors' contribution}

NKS involved in conception, data organization, data interpretation/analysis, draft preparation, critical review and approval, MG involved in conception, data acquisition, critical review of draft, approval, VG in involved critical review of draft, draft revision, approval, AM in involved conception, data collection, critical review of draft, approval.

\section{References}

I. Nabi G, Dinda AK, Dogra PN: Primary embryonal rhabdomyosarcoma of prostate in adults: diagnosis and management. Int Urol Nephrol 2002, 34:53I-534.

2. Dalal DD, Tongaonkar HB, Krishnamurthy S: Kulkarni JN. Embryonal rhabdomyosarcoma of prostate in an adult a diagnostic dilemma. Indian / Cancer 2000, 37:50-53.

3. Figueiredo AA, Brandão G, Dentz LC, Alves CM, Stroppa AE: Bilateral pulmonary alveolar infiltrate and prostate tumour in a 54-year-old man. Thorax 2007, 62:1002-1018.

4. Raney RB, Anderson JR, Barr FG, Donaldson SS, Pappo AS, Qualman SJ, Wiener ES, Maurer HM, Crist WM: Rhabdomyosarcoma and undifferentiated sarcoma in the first two decades of life: a selective review of intergroup rhabdomyosarcoma study group experience and rationale for Intergroup Rhabdomyosarcoma Study V.J Pediatr Hematol Oncol 200 I, 23:2 1 5-220.

5. Raney RB, Stoner JA, Walterhouse DO, Andrassy RJ, Donaldson SS, Laurie F, Meyer WH, Qualman SJ, Crist WM: Results of treatment of fifty-six patients with localized retroperitoneal and pelvic rhabdomyosarcoma: a report from The Intergroup Rhabdomyosarcoma Study-IV, 199I-1997. Pediatr Blood Cancer 2004, 42:618-625.

\section{Do you have a case to share?}

Submit your case report today

- Rapid peer review

- Fast publication

- PubMed indexing

- Inclusion in Cases Database

\section{Any patient, any case, can teach us something}

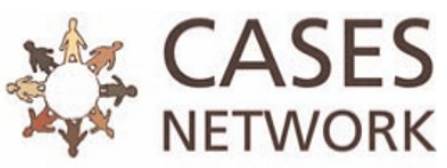

www.casesnetwork.com 